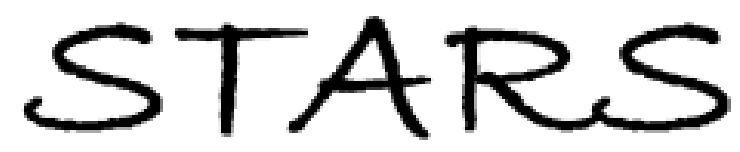

University of Central Florida

STARS

$1-1-1997$

\title{
Dynamic Band Unblocking And Leakage Two-Photon Absorption In Insb
}

M. P. Hasselbeck

University of Central Florida

E. W. Stryland

University of Central Florida

M. Sheik-Bahae

Find similar works at: https://stars.library.ucf.edu/facultybib1990

University of Central Florida Libraries http://library.ucf.edu

This Article is brought to you for free and open access by the Faculty Bibliography at STARS. It has been accepted for inclusion in Faculty Bibliography 1990s by an authorized administrator of STARS. For more information, please contact STARS@ucf.edu.

\section{Recommended Citation}

Hasselbeck, M. P.; Stryland, E. W.; and Sheik-Bahae, M., "Dynamic Band Unblocking And Leakage TwoPhoton Absorption In Insb" (1997). Faculty Bibliography 1990s. 1939.

https://stars.library.ucf.edu/facultybib1990/1939

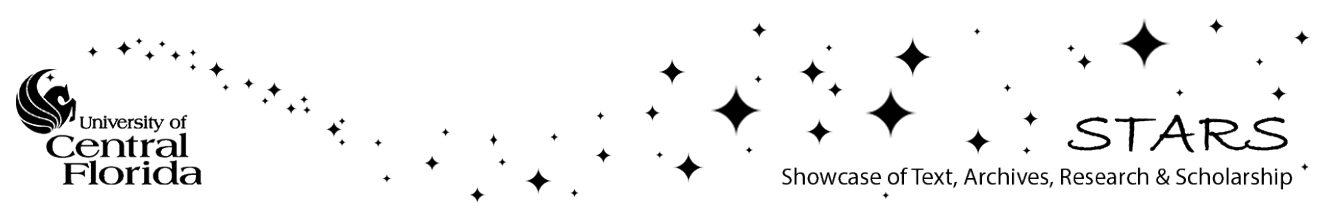




\title{
Dynamic band unblocking and leakage two-photon absorption in InSb
}

\author{
M. P. Hasselbeck ${ }^{*}$ and E. W. Van Stryland \\ Center for Research and Education in Optics and Lasers (CREOL), University of Central Florida, Orlando, Florida 32826 \\ M. Sheik-Bahae \\ Department of Physics and Astronomy, University of New Mexico, Albuquerque, New Mexico 87131
}

(Received 10 February 1997)

\begin{abstract}
An experimental demonstration of a dynamic decrease of the optical band gap of bulk $n$-InSb induced by picosecond, midinfrared laser pulses is reported. This occurs as a result of laser heating of the quiescent electron distribution by free-carrier absorption. The hot electrons vacate low-energy states near the conductionband minimum, unblocking terminal states for two-photon absorption across the band gap. This "leakage" two-photon absorption is detected as a consequence of changes to the refractive index and absorption coefficient of the semiconductor caused by photocarriers. The onset of dynamic band unblocking agrees with a calculation of laser-induced electron heating. [S0163-1829(97)03836-8]
\end{abstract}

\section{INTRODUCTION}

Some of the strongest nonlinear optical effects ever reported are the large changes of refractive index that occur in narrow-gap semiconductors when excited by a laser beam with photon energy very close to the band-gap energy. ${ }^{1}$ Laser light generates electron-hole pairs by direct excitation; if the rate of excitation exceeds that of recombination, an appreciable photocarrier density can be attained. At a sufficiently high concentration of conduction electrons, all available terminal states for cross-band transitions become filled giving rise to absorption bleaching - the dynamic BursteinMoss shift-and an associated large modulation of refractive index at the excitation wavelength. ${ }^{2}$ This effect has been used to construct a variety of optical switches. ${ }^{3}$ Although laser-induced band blocking was first observed in the materials InAsP and InGaAs, ${ }^{4}$ it is most pronounced in the narrow-gap semiconductors $\mathrm{HgCdTe},{ }^{5} \mathrm{InSb},{ }^{2,6-8}$ and InAs, ${ }^{9}$ where higher conduction-band curvature leads to band filling and bleaching at modest photocarrier densities often attainable with low power, cw lasers.

In this paper, we report observation of the converse effect: dynamic band unblocking. A quiescent electron distribution (such as provided by doping) completely fills states at and above the conduction-band edge. The laser frequency is chosen so that two-photon transitions are blocked $\left(2 \hbar \omega \cong E_{g}\right)$. At sufficiently high intensity, the laser beam removes the band-blocking condition by heating the quiescent electrons to higher-energy states in the conduction band. Electron heating is accomplished by linear free-carrier absorption of the infrared laser photons (photon-electron-phonon scattering). The chemical potential (Fermi level) is forced from a position above the conduction-band edge into the band gap. With terminal states for two-photon absorption in the conduction band open, cross-band generation of electron-hole pairs takes place. The presence of these photocarriers is readily detected by the excitation beam as a result of changes to both the refractive index and absorption coefficient of the semiconductor. Therefore, laser heating of conduction electrons effectively reduces the optical band-gap energy, giving rise to "leakage" two-photon absorption.

In the next section, band-unblocking experiments with InSb are described. A calculation of the laser-induced electron temperature is presented in Sec. III. The analysis shows that the optical absorption edge shifts by an amount sufficient to enable two-photon absorption at the laser irradiance levels encountered in the experiments. In Sec. IV, consideration is given to three-photon absorption, a possible competing process. We demonstrate that three-photon absorption is not important for our experimental conditions.

\section{EXPERIMENT}

Pulses of duration $\sim 125$ ps are obtained from an amplified TEA $\mathrm{CO}_{2}$ laser system configured in the optical freeinduction-decay arrangement. ${ }^{10}$ The grating-tuned TEA laser generates pulses at a wavelength of $10.6 \mu \mathrm{m}$ ( $\hbar \omega$ $=0.117 \mathrm{eV}$ ) and repetition frequency of $1 \mathrm{~Hz}$. Analysis of zero-background autocorrelation data indicates that $\sim 25 \%$ of the total pulse energy is contained in a second low power pulse of duration $\sim 400 \mathrm{ps}$ that follows the main pulse. The secondary pulse is an expected component of the freeinduction-decay pulse-shaping process. ${ }^{10}$ An uncoated, polished, single crystal of lightly $n$-doped $\operatorname{InSb}\left(N_{D}\right.$ $\sim 10^{16} \mathrm{~cm}^{-3}$; thickness $\cong 1 \mathrm{~mm}$ ) is mounted on the coldfinger of a closed-cycle helium refrigerator held at a temperature of $12-15 \mathrm{~K}$. A layer of indium between the sample and the coldfinger maintains good thermal contact. Energy deposition by the laser pulse is not sufficient to heat the semiconductor lattice appreciably $\left(\Delta T_{L}<1 \mathrm{~K}\right)$. Etalon effects are eliminated by orienting the crystal at an angle of $\sim 30^{\circ}$ from the optical axis ( $s$ polarization). Nonlinear absorption and refraction are measured using open and closed aperture $Z$ scans, respectively. ${ }^{11}$ The experimental arrangement is depicted in Fig. 1(a). The irradiance on the sample is varied by translating the cryostat through the focus of a positive lens telescope (measured beam waist: $\omega_{0}=124 \pm 3 \mu \mathrm{m}$ ).

Nonlinear absorption of $10.6 \mu \mathrm{m}$ laser light is found at both room temperature $\left(E_{g}=0.18 \mathrm{eV}\right)$ and $15 \mathrm{~K}\left(E_{g}\right.$ $=0.235 \mathrm{eV})$ in the same experimental setup. Normalized dif- 

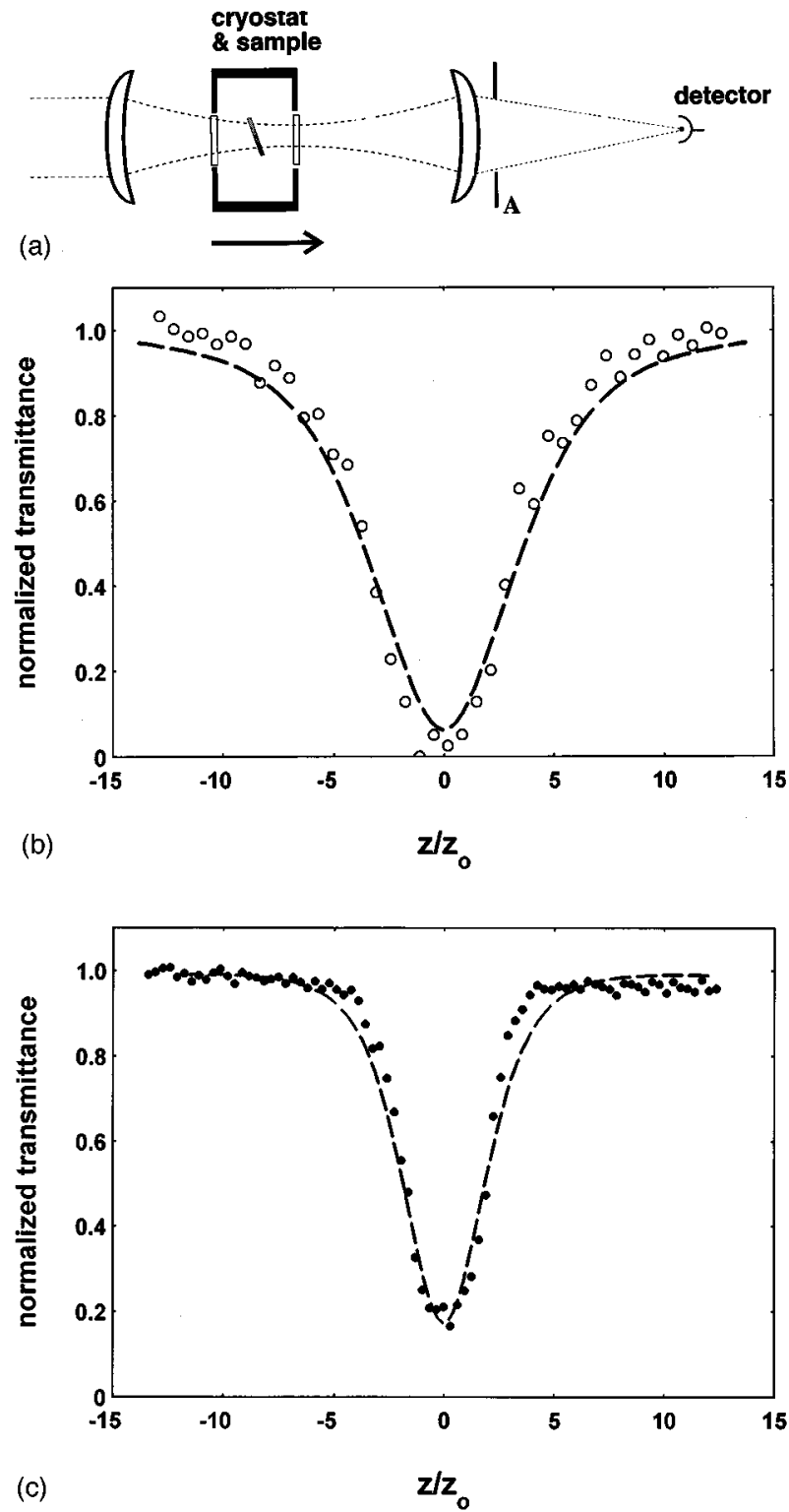

FIG. 1. (a) Z-scan experiment for obtaining nonlinear refraction and absorption. Nonlinear absorption is isolated by completely opening the aperture $(A)$. (b) Open aperture $Z$ scan of the InSb sample at $300 \mathrm{~K}$. Dashed curve obtained with 2PA model represented by Eqs. (1) and (2) using parameters from Table I. (c) Same experiment except the lattice temperature is $15 \mathrm{~K}$. Dashed curve generated by the 2PA model neglecting hot electrons $\left(K_{2}\right.$ $=0.1 \mathrm{~cm} / \mathrm{MW}$ ). Deviation of data and curve at $\left|z / z_{0}\right| \sim 3$ reveals the inadequacy of the simple 2PA model. The peak irradiance in both $Z$ scans is $\sim 70 \mathrm{MW} / \mathrm{cm}^{2}$.

ferential absorption obtained in a sequence of open aperture $Z$ scans at these two temperatures is shown in Figs. 1(b) and $1(c)$. As the sample is translated through focus of the Gaussian beam, the irradiance increases to a peak value of $\sim 70 \mathrm{MW} / \mathrm{cm}^{2}$ at the center of the scan $\left(z / z_{0}=0\right.$, where $z_{0}$ is the confocal beam parameter). The strong differential absorption observed at $300 \mathrm{~K}$ [Fig. 1(b)] is due to the wellknown and thoroughly studied two-photon absorption (2PA) process in InSb. ${ }^{12}$ The room-temperature data, which is noisy because of low signal levels, is analyzed with the usual model of laser beam propagation in a semiconductor in the
TABLE I. Parameters used in the calculation.

\begin{tabular}{|c|c|c|}
\hline \multicolumn{2}{|c|}{ LO phonon energy $\left(\hbar \omega_{\mathrm{LO}}\right)$} & $23 \mathrm{meV}$ \\
\hline \multicolumn{2}{|c|}{ Electron effective mass $\left(m^{*}\right)$} & $0.015 \mathrm{~m}$ \\
\hline \multicolumn{2}{|l|}{$\epsilon_{0}$} & 17.54 \\
\hline \multicolumn{2}{|l|}{$\epsilon_{\infty}$} & 15.68 \\
\hline \multicolumn{2}{|c|}{$K_{2}(300 \mathrm{~K})^{\mathrm{a}}$} & $2 \mathrm{~cm} / \mathrm{MW}$ \\
\hline \multicolumn{3}{|c|}{ Free carrier absorption cross section at $\lambda=10.6 \mu \mathrm{m}^{\mathrm{b}}$} \\
\hline \multicolumn{2}{|r|}{$300 \mathrm{~K}$} & $15 \mathrm{~K}$ \\
\hline$\sigma_{e}$ & $3 \times 10^{-17} \mathrm{~cm}^{2}$ & $1.9 \times 10^{-17} \mathrm{~cm}^{2}$ \\
\hline$\sigma_{h}$ & $8 \times 10^{-16} \mathrm{~cm}^{2}$ & $2 \times 10^{-15} \mathrm{~cm}^{2}$ \\
\hline
\end{tabular}

${ }^{\mathrm{a}}$ References 12 and 48 .

${ }^{\mathrm{b}}$ Reference 51 .

presence of 2PA generated photocarriers: ${ }^{13}$

$$
\begin{gathered}
\frac{d}{d z} I(r, z, t)=-K_{2} I^{2}-\left(\sigma_{e} N_{e}+\sigma_{h} N_{h}\right) I, \\
\frac{d N_{e}}{d t}=\frac{d N_{h}}{d t}=\frac{K_{2} I^{2}}{2 \hbar \omega},
\end{gathered}
$$

where $I$ is the temporally and spatially varying laser irradiance, $K_{2}$ is the 2PA coefficient, $\hbar \omega$ is the photon energy, $N_{e}$ and $N_{h}$ are the electron and hole concentrations, respectively, with $\sigma_{e, h}$ the infrared optical absorption cross sections. Recombination and diffusion of carriers can be ignored on the 100 ps time scale of the experiment. ${ }^{14-17}$ These equations are integrated numerically through the depth of the sample using the laser temporal profile and Gaussian beam cross section. The various parameters used to generate the dashed curve in Fig. 1(b) have all been obtained from the literature and are collected in Table I. The differential absorption is dominated by holes generated in the 2PA process $\left(\Delta N_{h} \sim 10^{15} \mathrm{~cm}^{-3}\right)$. The photogenerated holes dramatically increase the linear absorption of laser light [third term on the RHS of Eq. (1)] because of dipole-allowed transitions between the heavyand light-hole valence bands.

When the sample temperature is lowered to $15 \mathrm{~K}$, the semiconductor band gap expands, putting the two-photon energy just below the optical band edge $\left(2 \hbar \omega \cong E_{g}\right)$ while the electron chemical potential shifts to a position approximately $10 \mathrm{meV}$ above the band edge. The dashed curve in Fig. 1(c) is a fit to the $15-\mathrm{K}$ data near the center of the $Z$ scan using the 2PA model of Eqs. (1) and (2) with $K_{2}$ as a free parameter. Note the deviation of the data from the calculated curve in the wings of the $Z$ scan, where the irradiance is much lower than at the peak $\left(I<20 \mathrm{MW} / \mathrm{cm}^{2}\right)$. In the low irradiance regime (large values of $|z|$ ), the electron temperature remains close to that of the lattice, keeping terminal states for 2PA completely occupied. The simple 2PA model (which neglects hot carriers) overestimates the differential absorption here. At higher irradiance (smaller values of $|z|$ ), laser heating of electrons becomes significant, causing a redistribution to higher-energy conduction-band states, thereby unblocking terminal states for 2PA near the band edge. The dashed curve in Fig. 1(c) is generated with a 2PA coefficient of $K_{2}=0.1 \mathrm{~cm} / \mathrm{MW}$ and the appropriate parameters from Table I, but without any consideration of hot carriers. 


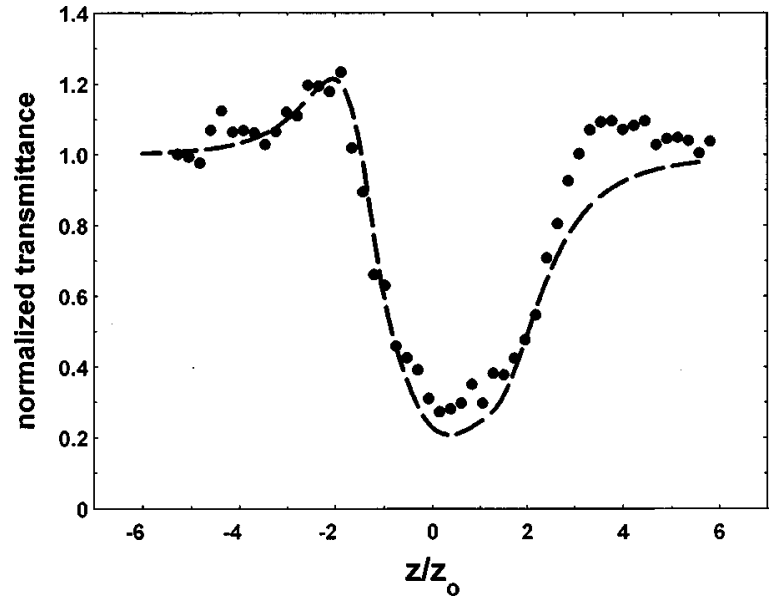

FIG. 2. $Z$ scan at $15 \mathrm{~K}$ using a partially transmitting aperture. Increased differential transmission at $z / z_{0} \sim-2$ is caused by plasma defocusing, followed by negative differential transmission due to photocarrier absorption $\left(z / z_{0}>-1\right)$. The dashed curve is fit to data that include refraction and absorption by photocarriers, but without consideration of carrier heating. Peak irradiance is $\sim 30 \mathrm{MW} / \mathrm{cm}^{2}$ at $z / z_{0}=0$.

When a partially obscuring aperture is placed in the far field of the laser beam before entering the detector, the $Z$ scan can sense changes to the semiconductor refractive index caused by self-focusing or defocusing. ${ }^{11}$ In the case of twophoton generated electron-hole pairs, far-field defocusing of the infrared beam takes place when the irradiance on the sample increases.

In Fig. 2, an apertured $Z$ scan (transmission $\cong 0.4$ ) of the InSb sample at $15 \mathrm{~K}$ reveals the expected defocusing behavior as the cryostat is translated though the focus of the Gaussian beam. Because of the dominant absorption caused by the photocarriers, the peak irradiance of the $Z$ scan is reduced to $\sim 30 \mathrm{MW} / \mathrm{cm}^{2}$ to make the differential refraction change clearer. Both defocusing (positive differential signal for $z / z_{0}<-1$ ) and absorption (negative differential signal for $\left.z / z_{0}>-1\right)$ are present in Fig. 2.

The data in Fig. 2 are analyzed with Eqs. (1) and (2) in a propagation code that includes a temporally and spatially varying refractive index in the semiconductor sample due to the electron-hole plasma: ${ }^{18,19}$

$$
\Delta n=\frac{2 \pi e^{2} \Delta N}{m^{*} \omega^{2} n_{0}} \frac{E_{g}^{2}}{E_{g}^{2}-\hbar \omega^{2}},
$$

where $e$ is the electronic charge, $m^{*}$ is the electron effective mass, $n_{0}$ is the linear refractive index, and $\Delta N$ is the photo- carrier density. For InSb at $15 \mathrm{~K}$ and a laser wavelength of $\lambda=10.6 \mu \mathrm{m}$, we have $\Delta n / \Delta N=1.1 \times 10^{-18} \mathrm{~cm}^{-3}$. The high irradiance data (small values of $\left|z / z_{0}\right|$ ) is fit with a $2 \mathrm{PA}$ coefficient of $K_{2}=0.09 \pm 0.01 \mathrm{~cm} / \mathrm{MW}$, as depicted by the dashed curve in Fig. 2. Note that the 2PA model described by Eqs. (1)-(3) again overestimates the nonlinear absorption (large positive values of $z / z_{0}$ ). The discrepancy is attributed to the presence of hot carriers, which have been ignored in the simple model. The value of $K_{2}$ used to fit the data in Fig. 2 agrees with the leakage 2PA coefficient extracted from the unapertured data in Fig. 1(c).

\section{CARRIER HEATING AND BAND UNBLOCKING}

A description of the electrons using temperature in a nonparabolic conduction band with Fermi-Dirac statistics is appropriate for the sample carrier concentration and time scale of the experiment. ${ }^{20}$ For a donor density of $N_{D}$ $\sim 10^{16} \mathrm{~cm}^{-3}$, the Fermi level resides $\sim 10 \mathrm{meV}$ above the conduction band edge when $T_{e}=T_{L}=15 \mathrm{~K}$. Longitudinaloptical phonon emission is the dominant energy loss mechanism in InSb when $T_{e}>20 \mathrm{~K} .{ }^{21}$ The characteristic time of LO phonon emission by hot electrons in InSb is a few picoseconds. Because the laser pulse duration is more than an order of magnitude longer, a quasi-steady-state analysis is used, i.e., the electron temperature is determined by the instantaneous value of the laser irradiance. By extrapolating the measurements of Grann et al., ${ }^{22}$ we find that the anharmonic decay of LO phonons in InSb occurs in 3-4 ps at 15 $\mathrm{K}$. Therefore, phonon reabsorption by the electrons (hot phonon effect) is neglected. The irradiance-dependent electron temperature is calculated using the following equation, in which energy deposited into conduction electrons is exactly balanced by LO phonon emission: ${ }^{23}$

$$
\sigma_{e}\left(T_{E}\right) I=\left\langle\frac{d E}{d t}\right\rangle_{\text {LO phonons }} .
$$

The point of balance defines the electron temperature for a given laser irradiance. A temperature-dependent electronic infrared free-carrier absorption cross section $\sigma_{e}$ is used in the calculation, discussed in more detail below. The computed energy-loss rate $\langle d E / d t\rangle$ accounts for both phonon emission and absorption, conduction-band nonparabolicity, and dynamic screening of the Fröhlich potential ${ }^{24-26}$ averaged over a Fermi-Dirac distribution at temperature $T_{e}$. Using the notation of Ref. 27, the dominant phonon emission component is

$$
\left\langle\frac{d E}{d t}\right\rangle_{\substack{\text { phonon } \\ \text { emission }}}=\frac{\left(m^{*} e\right)^{2} \hbar \omega_{\mathrm{LO}}}{\pi^{3} \hbar^{5} N_{e}} \int_{0}^{\infty} \frac{d \xi G(\xi)}{\xi} \int d \hbar \omega \operatorname{Im}\left(\frac{1}{\varepsilon(\xi, \omega)}\right)\left[N_{q}(\omega)+1\right] \int_{E_{\min }}^{\infty} d E(1+2 \alpha E) f(E)\left[1-f\left(E-\hbar \omega_{\mathrm{LO}}\right)\right]
$$

where $N_{q}$ is the Bose occupation factor, $f(E)$ is the Fermi function, $\alpha$ is the Kane nonparabolicity coefficient $\left(\alpha=1 / E_{g}\right),{ }^{28}$ $\hbar \omega_{\mathrm{LO}}$ is the optical phonon energy $(0.023 \mathrm{eV})$, and the lower limit of the inner integral is

$$
E_{\min }=\frac{1}{2 \alpha}\left[-1+\sqrt{1+2 \alpha k T_{e}\left(\frac{\hbar \omega_{\mathrm{LO}}}{2 k T_{e} \xi}+1\right)^{2}}\right] .
$$


The phonon absorption expression is obtained by replacing $N_{q}+1 \rightarrow N_{q}$ and $E \rightarrow E+\hbar \omega_{\mathrm{LO}}$ in the Fermi functions. The difference of these two components determines the aggregate energy loss rate. The function $G$ accounts for overlap of initial- and final-state electron wave functions in a nonparabolic conduction band. ${ }^{29}$ For a parabolic band, $G$ is unity. Analysis of the overlap function in a nonparabolic band has been performed by Fawcett et al. ${ }^{30}$ To first order in $\alpha$, their nonparabolic overlap factor is approximated as

$$
\begin{array}{ll}
G(\xi)=1-2 \alpha k T_{e} \xi & \text { for } \xi<\frac{1}{2 \alpha k T_{e}}, \\
G(\xi)=0 & \text { for } \xi>\frac{1}{2 \alpha k T_{e}},
\end{array}
$$

where the usual phonon momentum term $q$ is replaced by the dimensionless variable $\xi=(\hbar q)^{2} / 4 m^{*} k T_{e}$. The formulation of the overlap function in Eq. (6) defines a cutoff wave vector beyond which no phonon scattering takes place. Both parabolic $(G=1)$ and first-order nonparabolic overlap factors [Eq. (6)] are considered in the electron temperature calculation and bound a more exact treatment of the problem.
As will be seen, for the electron temperatures relevant for the band-unblocking experiment, a more sophisticated analysis of this overlap factor is not important.

The optical phonon frequency and electron-hole plasma frequency are comparable in our InSb sample, making a dynamic screening treatment of the Fröhlich potential appropriate. $^{31}$ The poles of the reciprocal dielectric function $\operatorname{Im}[1 / \varepsilon(\xi, \omega)]$ define the phonon mode of the coupled plasmon-phonon system. ${ }^{24}$ We have included conductionband nonparabolicity in the analysis of this momentum- and frequency-dependent dielectric function. At the electron density considered here $\left(10^{16} \mathrm{~cm}^{-3}\right)$, plasmons are largely Landau damped in the relevant region of reciprocal space, which is set by momentum conservation in the electron-phonon scattering process. An upper hybrid mode (i.e., phonon mode) exhibiting a weak frequency dependence is readily identified. The function $\varepsilon(\xi, \omega)$ must be evaluated for each electron temperature considered in the calculation.

In the limit of low electron density $\left(10^{14} \mathrm{~cm}^{-3}\right)$ and parabolic conduction band, the calculated LO phonon energy relaxation rate is found to agree with the well-known analytic formula $^{32}$

$$
\left\langle\frac{d E}{d t}\right\rangle=\sqrt{\frac{2 m^{*}}{\pi}} \frac{e^{2}\left(\hbar \omega_{\mathrm{LO}}\right)^{3 / 2}}{\hbar^{2}}\left[\frac{1}{\varepsilon_{\infty}}-\frac{1}{\varepsilon_{0}}\right] \frac{\exp \left(x_{0}-x_{e}\right)-1}{\exp \left(x_{0}\right)-1} \sqrt{x_{e}} \exp \left(\frac{x_{e}}{2}\right) K_{0}\left(\frac{x_{e}}{2}\right)
$$

where

$$
x_{0}=\frac{\hbar \omega_{\mathrm{LO}}}{k T_{L}} \text { and } x_{e}=\frac{\hbar \omega_{\mathrm{LO}}}{k T_{e}} .
$$

The hot electron cooling rates are plotted in Fig. 3. Shown is the full calculation with the two overlap factors for InSb at an electron concentration of $10^{16} \mathrm{~cm}^{-3}$ and a lattice temperature of $15 \mathrm{~K}$. The analytic expression given by Eq. (7) is plotted for comparison. We find that screening is not important at this carrier density. Deviation of the simple model given by Eq. (7) and the full treatment for the case of $G$ $=1$ is due to nonparabolicity in the density of states.

Temperature-dependent free-carrier absorption (FCA) by electrons has been observed previously at midinfrared wavelengths. ${ }^{33,34}$ The behavior of FCA with increasing electron temperature is evaluated following the calculation outlined by Elsaesser et al.; ${ }^{34}$ we modify this analysis to include dynamic screening and conduction-band nonparabolicity, but neglect hot phonons for reasons given previously. We assume momentum conservation is obtained entirely by LO phonon scattering. Results of this calculation, for two different treatments of the wave-function overlap factor $(G)$, are shown in Fig. 4 for an electron concentration of $10^{16} \mathrm{~cm}^{-3}$. Only the relative temperature dependence of FCA is shown. To evaluate laser-induced changes to the electron temperature, the previously measured linear cross section (Table I) is scaled according to the curves in Fig. 4 in the hightemperature regime. ${ }^{35}$
The remaining task is to solve the transcendental balance equation [Eq. (4)] for the electron temperature by combining the results in Figs. 3 and 4 for two different treatments of the overlap function $(G)$. Results are presented in Fig. 5. Higher electron temperature is attained in the calculation with the

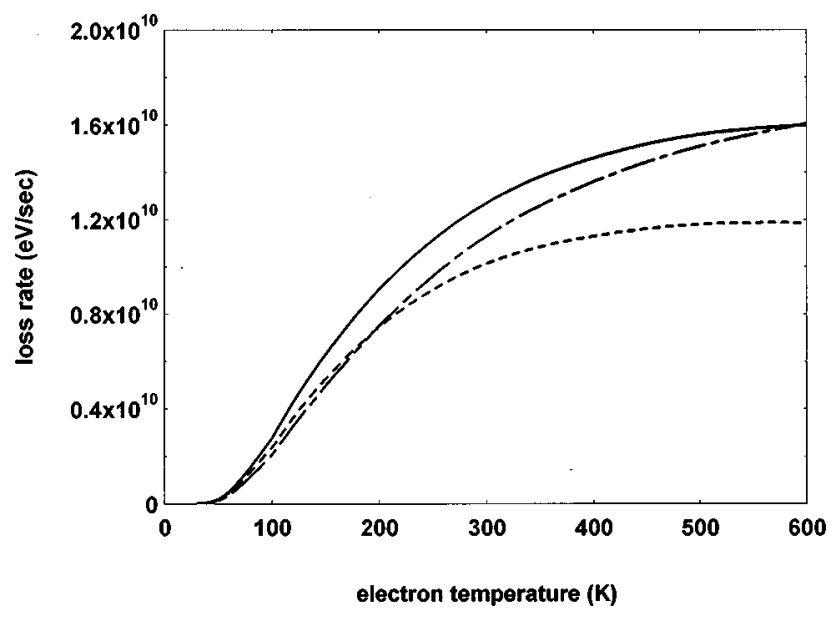

FIG. 3. Electron-energy-loss rate to due LO phonon emission in InSb at a lattice temperature of $15 \mathrm{~K}$ and donor density of $10^{16} \mathrm{~cm}^{-3}$. Shown are full calculations with unity overlap factor (solid curve), inclusion of a nonparabolic overlap factor described by cutoff wave vector (dotted curve), and simple MaxwellBoltzmann model that neglects nonparabolicity and screening (dash-dotted curve). 


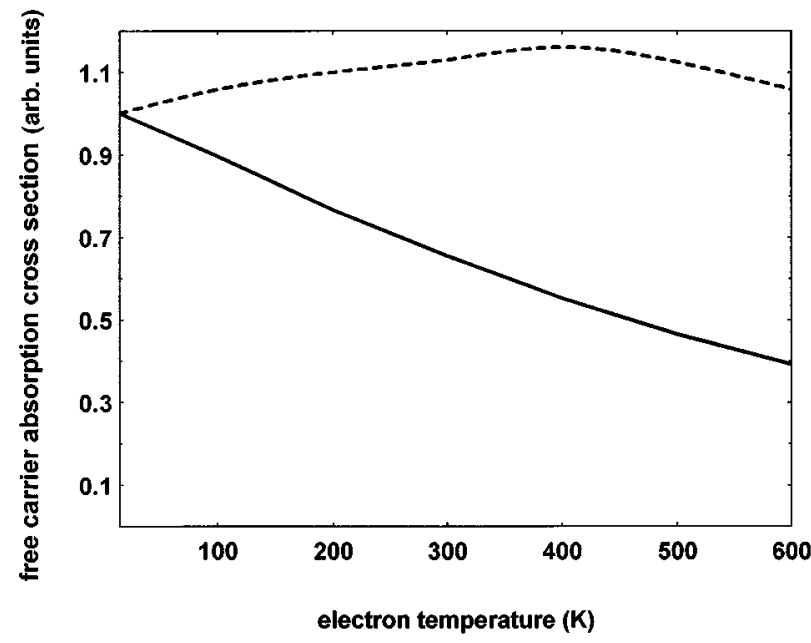

FIG. 4. Absorption cross section of hot electrons at $\lambda$ $=10.6 \mu \mathrm{m}$, lattice temperature of $15 \mathrm{~K}$, and donor density of $10^{16} \mathrm{~cm}^{-3}$. Two treatments of wave-function overlap are shown: unity overlap (dotted line) and cutoff wave-vector approximation (solid line). The curves are normalized at $T_{L}=T_{e}=15 \mathrm{~K}$.

unity overlap factor as the irradiance exceeds $50 \mathrm{MW} / \mathrm{cm}^{2}$. Although cooling is less efficient in the nonparabolic treatment of $G$, the cross section for electron excitation is comparatively smaller (Fig. 4), which leads to lower temperature. In the irradiance range of interest $\left(20-70 \mathrm{MW} / \mathrm{cm}^{2}\right)$, both calculations give essentially the same result. Also plotted in Fig. 5 is the chemical potential of the electrons relative to the conduction-band edge as a function of laser irradiance, using the heating calculation depicted by the solid curve. The chemical potential is found to shift below the band edge at an irradiance of $\sim 30 \mathrm{MW} / \mathrm{cm}^{2}$. This roughly defines the point where sufficient band unblocking permits the $2 \mathrm{PA}$ process to take place, in agreement with the experimentally observed "threshold" for the onset of photocarrier generation in Fig. $1(\mathrm{c})$.

Our calculations show that as the carrier concentration builds up due to photoexcitation, laser heating of electrons

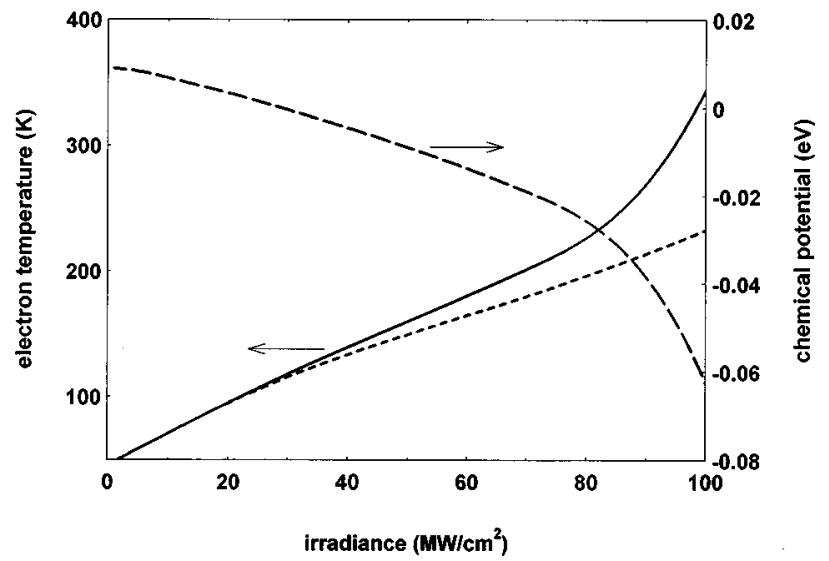

FIG. 5. Laser heating of conduction electrons $\left(N_{e}\right.$ $=10^{16} \mathrm{~cm}^{-3}$ ) at $\lambda=10.6 \mu \mathrm{m}$ for the two overlap functions: unity overlap (solid curve) and cutoff approximation (dotted curve). Also shown is the electron chemical potential relative to the conduction band edge. A shift below the band edge at $\sim 30 \mathrm{MW} / \mathrm{cm}^{2}$ indicates the approximate onset of $2 \mathrm{PA}$. maintains free terminal states for continued 2PA at increasing irradiance. This is in contrast to an earlier suggestion that discarded the possibility of $2 \mathrm{PA}$ in a similar experiment because of band blocking by cold electrons, i.e., a dynamic Burstein-Moss shift. ${ }^{36}$ It is also important to point out that our analysis indicates the electrons are not hot enough to initiate significant impact ionization scattering. ${ }^{37}$ The role of impact ionization in this and other laser experiments with $\mathrm{InSb}$ is discussed in Sec. IV.

We stress the availability of terminal states for twophoton transitions at and just below the conduction-band edge. There is no donor freezeout in InSb and the small effective mass leads to an overlap of donor electron orbitals that causes a continuum of states at and below the conduction band edge, even in relatively pure material. ${ }^{38}$ Using the theory of Stern and Talley, ${ }^{39}$ we estimate that this continuum extends approximately $4-9 \mathrm{meV}$ below the bottom of the conduction band in InSb at our sample donor density. When the electrons are cold $\left(T_{e} \approx T_{L}\right)$ these states are completely occupied, leading to an optical band gap of $0.24 \mathrm{eV}$ at $15 \mathrm{~K}$. The theory neglects the random distribution of impurities however, which causes a tail in the density of states below the band edge. Absorption involving band-tail states, which is also important for analysis of band blocking involving single-photon transitions, is not well understood. ${ }^{2}$ Here we deem it sufficient to determine the threshold irradiance for band unblocking by tracking the position of the chemical potential. A complete description of the $Z$-scan data in Figs. 1 (c) and 2 (at, above, and below the band-unblocking irradiance) is beyond the scope of this paper.

We comment on the leakage 2PA coefficient deduced from our data at $T_{L}=15 \mathrm{~K}\left(K_{2}=0.1 \mathrm{~cm} / \mathrm{MW}\right)$. The established scaling law for 2PA (Refs. 40-42) can be used to compare it with the room-temperature result at $10.6 \mu \mathrm{m}$ $\left(K_{2}=2 \mathrm{~cm} / \mathrm{MW}\right)$. Specifically, we can use the scaling of 2PA to deduce the "effective" optical band gap in the hot electron regime. Denoting the effective band gap as $E_{\text {hot }}$, we have

$$
\begin{aligned}
\frac{K_{2}(300 \mathrm{~K})}{K_{2}(15 \mathrm{~K})} & =\frac{2 \mathrm{~cm} / \mathrm{MW}}{0.1 \mathrm{~cm} / \mathrm{MW}} \\
& =\sqrt{E_{\mathrm{hot}} / E_{g}(300 \mathrm{~K})}\left[\frac{2 \hbar \omega-E_{g}(300 \mathrm{~K})}{2 \hbar \omega-E_{\mathrm{hot}}}\right]^{3 / 2},
\end{aligned}
$$

which leads to $E_{\text {hot }}=0.23 \mathrm{eV}$. Given that the optical band gap of InSb is measured to be $E_{g}(15 \mathrm{~K})=0.24 \mathrm{eV}$ in the absence of hot electrons, we estimate a reduction of the band-gap energy by an amount $\Delta E \approx 10 \mathrm{meV}$ in our experiment. This is the approximate amount of band unblocking, corresponding to an electron temperature of $T_{e}=\Delta E / k$ $\cong 115 \mathrm{~K}$, which is consistent with the electron heating calculations presented in this section. This is a crude analysis, however, because it neglects the reduction in density of states as a result of band tailing. Scaling of 2PA represented by Eq. (8) only provides an order of magnitude estimate for the increase of electron temperature.

We note the distinction between leakage 2PA and hotelectron-assisted absorption described by Ryvkin. ${ }^{43}$ Applied to the present InSb experiments, Ryvkin's absorption mecha- 
nism is manifest as a four-body process involving two electrons and two photons. The two-photon energy deficit $\Delta E$ $=2 \hbar \omega-E_{g}$ is supplied by a hot electron that causes scattering from a virtual state within the semiconductor bandgap into the conduction band. In contrast, leakage $2 \mathrm{PA}$ is a threebody process (electron-photon-photon) that terminates in real states made available only when the conduction electrons are sufficiently hot.

\section{THREE-PHOTON ABSORPTION}

The fact that a simple 2PA model (i.e., a model neglecting hot carriers) overestimates the differential absorption seen in our unapertured $Z$-scan data in the low irradiance regime [Fig. 1(c)] indicates that higher-order nonlinear absorption is taking place. An obvious alternative to the hot carrierinduced leakage 2PA explanation proposed here is threephoton absorption (3PA). To investigate this possibility, we assume 2PA remains blocked for the entire laser pulse and apply instead a 3PA model. ${ }^{44}$ The open and closed aperture data are fit with a 3PA coefficient of $K_{3}=0.04$ $\pm 0.02 \mathrm{~cm}^{3} / \mathrm{MW}^{2}$. This number, however, is considerably higher than predicted by available theories. Applying the dressed-wave-function formulation of Keldysh, we obtain $K_{3}=2.7 \times 10^{-3} \mathrm{~cm}^{3} / \mathrm{MW}^{2}$ for our InSb experiment. ${ }^{45} \mathrm{~A}$ similar treatment by Brandi and de Araujo yields $K_{3}=2.4$ $\times 10^{-3} \mathrm{~cm}^{3} / \mathrm{MW}^{2}{ }^{41}$ Wherrett's analysis of 3PA uses thirdorder perturbation with a simple two-band model and gives $K_{3}=3.8 \times 10^{-3} \mathrm{~cm}^{3} / \mathrm{MW}^{2}{ }^{40}$

Although 3PA appears to play a negligible role in the interpretation of the present data, it may be important in the absence of hot carriers. Measurements have been made with cryogenic InSb $(2-20 \mathrm{~K})$ using much lower power $\mathrm{CO}_{2}$ laser pulses $(\lambda=10.6 \mu \mathrm{m})$ with durations ranging from $1 \mathrm{~ns}$ to 1 $\mu \mathrm{s}{ }^{15,46-49}$ Nonlinear absorption of laser light was found to occur when the irradiance was in the range $1-3 \mathrm{MW} / \mathrm{cm}^{2}$. Because $2 \hbar \omega<E_{g}, 2 \mathrm{PA}$ was ruled out and the differential absorption behavior attributed to laser-induced impact ionization. ${ }^{23,47,49}$ Our model of carrier heating (Fig. 5), which is time independent for laser pulses longer than $10 \mathrm{ps,} \mathrm{indi-}$ cates the electrons are negligibly heated at this level of excitation. Previous analyses have incorporated a free-electron absorption cross section that is much larger than the measured value (Table I) producing a substantial overestimate of the electron temperature. In their experiments using 1-ns laser pulses, Jamison and Nurmikko observed a photocarrier density estimated to be $\Delta N \sim 10^{15} \mathrm{~cm}^{-3}$ at an irradiance of $2-3 \mathrm{MW} / \mathrm{cm}^{2} .{ }^{49}$ Neglecting recombination, this result leads to a 3PA coefficient of $K_{3}=2-7 \times 10^{-3} \mathrm{~cm}^{3} / \mathrm{MW}^{2}$, which is consistent with the theoretical predictions given above. The conclusion is that 3PA at $\lambda=10.6 \mu \mathrm{m}$ can be detected in $\mathrm{InSb}$ provided the conduction electrons remain close to the lattice temperature and block 2PA. Carrier heating may be unavoidable in experiments with high intensity picosecond pulses. Although 3PA is present in the band-unblocking experiments reported here, it does not generate a sufficient photocarrier density in the $\sim 100 \mathrm{ps}$ time scale of the experiment. A much stronger effect (i.e., leakage 2PA) is needed to explain our data.

To confirm that 3PA is indeed negligible in the picosecond $Z$-scan measurements, a pump-probe experiment is performed using 125-ps $\mathrm{CO}_{2}$ laser pulses. The peak pump irra-

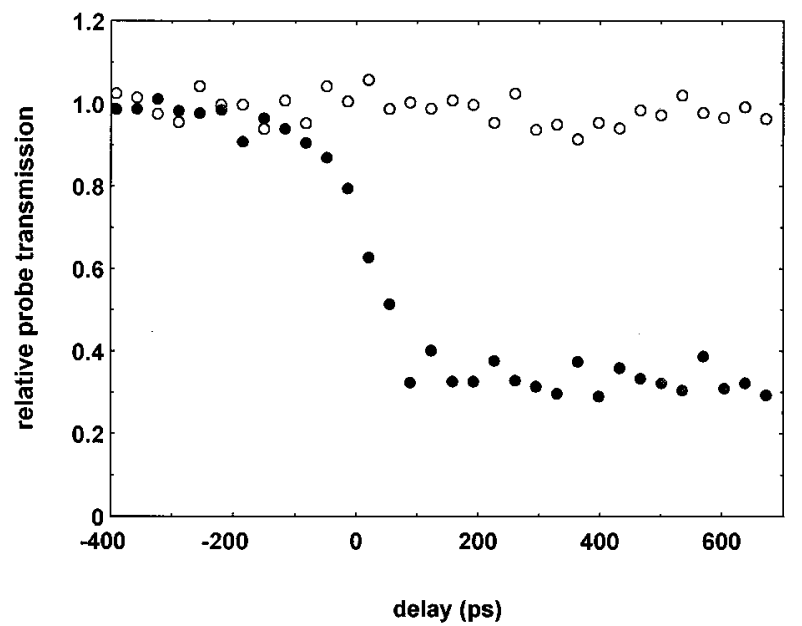

FIG. 6. Pump-probe data obtained with the InSb sample at $15 \mathrm{~K}$ and $\lambda=10.6 \mu \mathrm{m}$ (solid points). Increased differential absorption is caused by photogenerated holes. Lack of additional absorption near zero delay indicates the holes have minimal excess kinetic energy and are unlikely to have originated from 3PA. Open circles are obtained with the pump beam blocked, illustrating measurement noise. Pump irradiance is $\sim 30 \mathrm{MW} / \mathrm{cm}^{2}$.

diance is $\sim 30 \mathrm{MW} / \mathrm{cm}^{2}$, polarized orthogonal to the probe to eliminate complications from coherent wave mixing. ${ }^{50} \mathrm{Be}-$ cause of signal-to-noise limitations, the irradiance of the probe pulse is only about 8 times weaker than the pump. Time-resolved absorption data is presented in Fig. 6. The pump pulse creates photocarriers that recombine on a time scale longer than the measurement ( $>1 \mathrm{~ns})$. Photoexcited holes are monitored by the probe pulse via dipole-allowed transitions between the heavy- and light-hole valence bands. Intervalence-band absorption is two orders of magnitude larger than intraconduction band free-carrier absorption at $10.6 \mu \mathrm{m} .{ }^{51}$ Therefore, we time resolve changes to the heavyhole population and temperature. The important observation is the lack of additional absorption around zero delay. Enhanced absorption is expected at zero time delay if the carriers are created by 3PA. In 3PA, excess kinetic energy is given to the photocarriers in an amount $\Delta E=3 \hbar \omega-E_{g}$ $\cong 110 \mathrm{meV} .{ }^{52}$ Hot holes would rapidly accumulate in the heavy-hole band (thermalization occurs in $<1$ ps) (Ref. 53) and increase the population in the lower states of the intervalence-band transitions, leading to an enhanced absorption "spike" at zero delay. Enhanced absorption caused by this effect has been seen in previous pump-probe experiments with InAs $\left(\hbar \omega<E_{g}\right)$ as the result of two-photon absorption ${ }^{33}$ and three-photon excitation. ${ }^{44}$ When the laser wavelength is tuned to make $m \hbar \omega \approx E_{g}$ where $m$ is an integer, the absorption spike around zero delay disappears. ${ }^{33} \mathrm{Ab}$ sence of such an absorption feature in Fig. 6 indicates that the photocarriers have minimal excess kinetic energy, consistent with two-photon excitation close to the band-gap energy. For all the above reasons, we discard 3PA in the interpretation of our data.

In a study with a similar laser system operating at $\lambda$ $=10.6 \mu \mathrm{m}$, Sheik-Bahae et al. reported 3PA of 65 ps pulses by heavily doped $n$-InSb at room temperature ${ }^{54}$ Their InSb sample was doped to a density of $1.5 \times 10^{18} \mathrm{~cm}^{-3}$, where significant band blocking by the degenerate carrier distribu- 


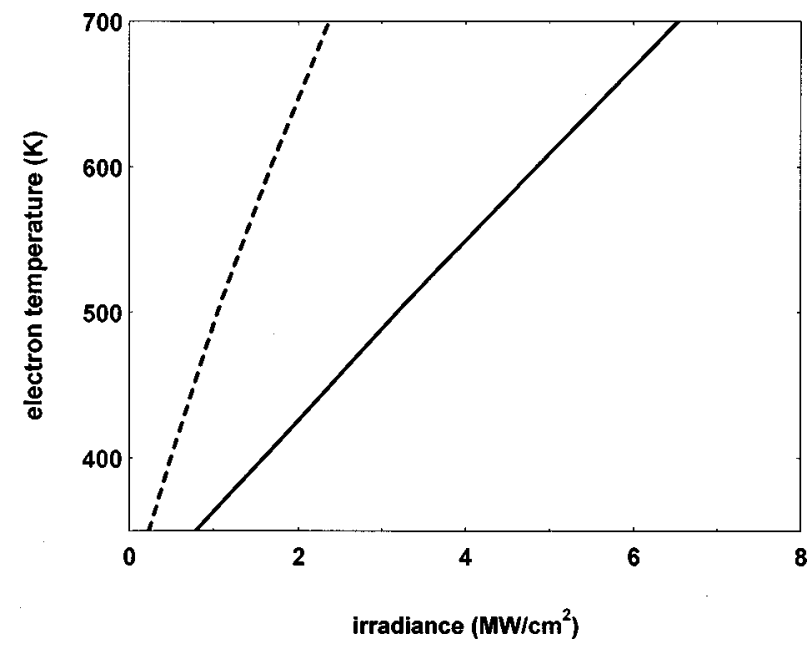

FIG. 7. Calculated laser heating of electrons in heavily $n$-doped InSb $\left(N_{D}=1.5 \times 10^{18} \mathrm{~cm}^{-3}\right)$ at a lattice temperature of $300 \mathrm{~K}$, relevant for the experiment of Ref. 54. Both unity overlap (solid line) and the cutoff wave-vector approximation (dotted line) are considered. A much higher electron temperature is predicted for this experiment because LO phonon emission is significantly screened.

tion occurs. ${ }^{55,56}$ This static Burstein-Moss shift causes the room-temperature optical band gap to increase from $0.18 \mathrm{eV}$ at low density $\left(N_{e}<5 \times 10^{17} \mathrm{~cm}^{-3}\right)$ to about $0.29 \mathrm{eV}$ when $N_{e}=1.5 \times 10^{18} \mathrm{~cm}^{-3}$. The optical band gap is therefore greater than $2 \hbar \omega=0.234 \mathrm{eV}$, making two-photon transitions energetically impossible in the absence of band unblocking. Increased differential absorption was observed to commence at an irradiance of $10-15 \mathrm{MW} / \mathrm{cm}^{2}$ inside the sample. The nonlinear behavior was attributed to free-carrier absorption by electron-hole pairs created in three-photon transitions across the bandgap. Their deduced 3PA coefficient of $K_{3}$ $=0.2 \mathrm{~cm}^{3} / \mathrm{MW}^{2}$ is also significantly higher than theoretical estimates. The dressed wave-function calculation of Keldysh yields $K_{3}=4.8 \times 10^{-3} \mathrm{~cm}^{3} / \mathrm{MW}^{2}$ (Ref. 45) and that of Brandi and de Araujo gives $K_{3}=7.2 \times 10^{-3} \mathrm{~cm}^{3} \mathrm{MW}^{2} .41$ Wherrett's third-order perturbation formula leads to $K_{3}$ $=3.1 \times 10^{-3} \mathrm{~cm}^{3} / \mathrm{MW}^{2} .{ }^{40}$ Wherrett also provides a material scaling relation for multiphoton absorption that allows us to estimate $K_{3}$ for InSb based on our measurements of 3PA with room-temperature InAs in the same experimental system. $^{44}$ This gives $K_{3} \approx 1.8 \times 10^{-3} \mathrm{~cm}^{3} / \mathrm{MW}^{2}$ for roomtemperature InSb at $\lambda=10.6 \mu \mathrm{m}$. Here again, estimates for the 3PA rate suggest it is far too weak to explain the observed nonlinear absorption.

Using the analysis presented in Sec. III, we find that hot carrier conditions $\left(T_{e}>T_{L}\right)$ may be expected for the conditions of the $n$-InSb experiment in Ref. 54. The electronenergy-loss rate due to LO phonon emission and the temperature-dependent FCA cross section are determined for heavily doped $n$-InSb. There is significant screening of small wave-vector phonons leading to a reduction of the cooling rate compared to lightly doped material. The results of this calculation are shown in Fig. 7, where the abscissa corresponds to irradiance inside the sample, i.e., corrected for Fresnel reflection. For both treatments of the overlap integral, the analysis shows that appreciable carrier heating takes place in the experiment reported in Ref. 54. We can assess the possibility of leakage 3PA using the data of Hrostowski et al. ${ }^{56}$ which reveal the optical absorption edge as a function of carrier density in room-temperature InSb. These measurements were made for quiescent conditions, with the electron temperature equal to the lattice temperature. Assuming that the lattice remains unperturbed by the high irradiance laser pulse however, the interpretation of Burstein is still valid for hot carrier conditions. ${ }^{55}$ The Fermi level $\left(E_{F}\right)$ is calculated as a function of density assuming a nonparabolic density of states. The energy $E_{F}$ is compared to the maximum energy for band blocking deduced from the linear absorption spectroscopy data. From the data of Ref. 56, the optical band gap $E_{0}$ is found to track the Fermi level following the approximate relationship

$$
E_{0} \approx E_{F}\left(T_{e}\right)-\frac{3}{2} k T_{e},
$$

where $E_{0}$ and $E_{F}$ are defined with respect to the valenceband edge. ${ }^{57}$ Our estimate shows that 2PA becomes energetically possible in heavily doped InSb $\left(N=1.5 \times 10^{18} \mathrm{~cm}^{-3}\right)$ as the electron temperature approaches $600 \mathrm{~K}$. As revealed by Fig. 7, this amount of heating can be expected for the experiment in question. The conclusion is that nonlinear absorption ascribed to 3PA reported in Ref. 54 may be reinterpreted as leakage 2PA. The primary issue is that heavy doping screens the Fröhlich potential allowing the electrons to acquire a temperature well above the lattice when exposed to picosecond infrared laser pulses, leading to conditions favorable for band unblocking.

We compare our work with similar experiments of Schwartz et al. $^{36}$ They measured the transmission of an InSb sample $\left(T_{L}=20 \mathrm{~K}\right)$ using variable duration laser pulses (8-60 ps) at a wavelength of $10.6 \mu \mathrm{m}$. Increased infrared absorption was observed at irradiance levels comparable to those in the present experiment due to the generation of photocarriers. We cannot, however, fit their data using analyses based on 2PA, 3PA, or their proposed impact ionization mechanism. Our calculation indicates carriers are not sufficiently heated to initiate impact ionization when the laser irradiance is less than $100 \mathrm{MW} / \mathrm{cm}^{2} .{ }^{37}$ Moreover, previous studies of the dynamics of impact ionization in InSb using pulsed electric fields show that it evolves on a time scale in excess of $1 \mathrm{~ns}$ and is therefore unlikely to be important in experiments with picosecond laser pulses. ${ }^{58-60}$ Schwartz et al. also report measurements at 300 and $77 \mathrm{~K}$ where 2PA is known to be operative $\left(2 \hbar \omega>E_{g}\right)$. We are likewise unable to fit this data with a model based on 2PA and suspect that only the central portion of the laser beam was collected by their detector.

\section{CONCLUSIONS}

By exploiting the sharp conduction-band curvature and relatively large absorption cross section of free electrons in the midinfrared, dynamic band unblocking has been demonstrated with InSb. A picosecond laser pulse heats the free electrons to a temperature much greater than the $15 \mathrm{~K}$ lattice temperature, causing a reduction of the effective optical band gap. This occurs because hot electrons vacate low-energy states in the conduction band. The reduction of band gap enables two-photon absorption (i.e., leakage two-photon absorption) and the concomitant generation of photocarriers. 
Photocarriers change both the semiconductor refractive index and linear absorption at the excitation laser frequency. Loss of infrared photons to the two-photon absorption process is a negligible component of the detected signals; direct absorption by generated holes and defocusing by the electron-hole plasma dominate the differential transmission measurements. The irradiance at which leakage two-photon absorption commences agrees with the calculated shift of the Fermi level associated with the laser-heated electrons.

There are four important factors that allow demonstration of this dynamic band-unblocking effect: (1) sharp curvature of the InSb conduction band $\left(m^{*}=0.015 \mathrm{~m}\right)$, (2) a comparatively large infrared absorption cross section for electrons, (3) a relatively high two-photon excitation rate in narrowgap semiconductors, and (4) dipole-allowed transitions between the heavy- and light-hole valence bands at the laser frequency. The first condition leads to a relatively small density of states near the conduction-band edge so that changes to the electron temperature and/or density significantly alter the position of the Fermi level. Second, a large electronic free-carrier absorption cross section allows the electrons to be heated at modest laser power $\left(<100 \mathrm{MW} / \mathrm{cm}^{-2}\right)$. The remaining two factors provide for efficient generation and detection of photocarriers, respectively, when the bandunblocking condition is attained. In essence, two-photon absorption serves to indicate a reduction of the Fermi level that accompanies an increase in electron temperature.

We comment briefly on the possibility of using band un-

*Present address: Max Born Institute for Nonlinear Optics and Ultrafast Spectroscopy, Berlin, Germany. Permanent address: USAF Phillips Laboratories, Albuquerque, New Mexico.

${ }^{1}$ A. Miller, D. A. B. Miller, and S. D. Smith, Adv. Phys. 30, 697 (1981).

${ }^{2}$ D. A. B. Miller, C. T. Seaton, M. E. Prise, and S. D. Smith, Phys. Rev. Lett. 47, 3 (1981).

${ }^{3}$ H. M. Gibbs, Optical Bistability: Controlling Light with Light (Academic, Orlando, 1985).

${ }^{4}$ J. F. Reintjes, J. C. McGroddy, and A. E. Blakeslee, J. Appl. Phys. 46, 879 (1975).

${ }^{5}$ J. R. Hill, G. Parry, and A. Miller, Opt. Commun. 43, 151 (1982).

${ }^{6}$ A. V. Nurmikko, Opt. Commun. 16, 365 (1976).

${ }^{7}$ P. Lavallard, R. Bichard, and C. Benoit a la Guillaume, Phys. Rev. B: Solid State 16, 2804 (1977).

${ }^{8}$ T. Elsaesser, H. Lobentanzer, and W. Kaiser, Appl. Phys. Lett. 47, 1190 (1985).

${ }^{9}$ C. D. Poole and E. Garmire, IEEE J. Quantum Electron. 21, 1370 (1985).

${ }^{10}$ E. Yablonovitch and J. Goldhar, Appl. Phys. Lett. 25, 580 (1974).

${ }^{11}$ M. Sheik-Bahae, A. A. Said, T. H. Wei, D. J. Hagan, and E. W. Van Stryland, IEEE J. Quantum Electron. 26, 760 (1990).

${ }^{12}$ M. Sheik-Bahae and H. S. Kwok, IEEE J. Quantum Electron. 23, 1974 (1987).

${ }^{13}$ E. W. Van Stryland, H. Vanherzeele, M. A. Woodall, M. J. Soileau, A. L. Smirl, S. Guha, and T. F. Boggess, Opt. Eng. (Bellingham) 24, 613 (1985).

${ }^{14}$ A. F. Gibson, M. J. Kent, and M. F. Kimmitt, J. Phys. D 1, 149 (1968).

${ }^{15}$ H. J. Fossum, W. S. Chen, and B. Ancker-Johnson, Phys. Rev. B 8, 2857 (1973). blocking to make a fast optical switch. A problem that has limited the usefulness of a switch based on band blocking in a passive semiconductor is the slow recombination rate of photocarriers. ${ }^{3}$ The switch recovery speed is intrinsically tied to this carrier recombination rate. In principle, a switch based strictly on carrier temperature does not have to involve photocarriers (in contrast to the present experiment). Low loss nonlinear refraction can be envisioned, since hot-carrierinduced changes to the absorption will manifest in changes to the refractive index. ${ }^{61}$ Therefore, the optical properties of the semiconductor could be modulated on a time scale limited by the dynamics of heating and cooling of carriers, which is of the order of a few picoseconds. In practice, however, multiphoton absorption is likely to be an issue because it will become important at irradiance levels comparable to that needed for carrier heating. ${ }^{62}$ Carrier generation can be avoided by using shorter pulses and extending the laser wavelength deeper into the infrared.

\section{ACKNOWLEDGMENTS}

The authors acknowledge helpful discussions with Professor M. Johnson, Professor T. Elsaesser, and Professor D. Ferry on various aspects of this work. We would also like to thank Dr. A. Said for supplying the propagation code used to analyze Fig. 2. M.P.H. acknowledges the support of Hughes Aircraft Company. This research was funded in part by NSF Grant No. 9510046.

${ }^{16}$ A. M. Johnston, C. R. Pidgeon, and J. Dempsey, Phys. Rev. B 22, 825 (1980)

${ }^{17}$ V. Chazapis, H. A. Blom, K. L. Vodopyanov, A. G. Norman, and C. C. Phillips, Phys. Rev. B 52, 2516 (1995).

${ }^{18}$ D. H. Auston, S. McAfee, C. V. Shank, E. P. Ippen, and O. Teschke, Solid-State Electron. 21, 147 (1980).

${ }^{19}$ A. A. Said, M. Sheik-Bahae, D. J. Hagan, T. H. Wei, J. Wang, J. Young, and E. W. Van Stryland, J. Opt. Soc. Am. B 9, 405 (1992).

${ }^{20}$ J. A. Kash and J. C. Tsang, in Light Scattering in Solids VI, edited by M. Cardona and G. Guntherodt (Springer-Verlag, Berlin, 1991).

${ }^{21}$ H. Kahlert and G. Bauer, Phys. Rev. B 7, 2670 (1973).

${ }^{22}$ E. D. Grann, K. T. Tsen, and D. K. Ferry, Phys. Rev. B 53, 9847 (1996).

${ }^{23}$ R. B. James, J. Appl. Phys. 54, 3220 (1983).

${ }^{24}$ M. Kim, A. Das, and S. Senturia, Phys. Rev. B 18, 6890 (1978).

${ }^{25}$ J. Young, N. Henry, and P. Kelly, Solid-State Electron. 32, 1567 (1989).

${ }^{26}$ J. Young, P. Kelly, and N. Henry, Solid State Commun. 78, 343 (1991).

${ }^{27}$ K. Seeger, Semiconductor Physics (Springer-Verlag, New York, 1989).

${ }^{28}$ E. O. Kane, J. Phys. Chem. Solids 1, 249 (1957).

${ }^{29}$ B. K. Ridley, Quantum Processes in Semiconductors (Clarendon, Oxford, 1988).

${ }^{30}$ W. Fawcett, D. A. Boardman, and S. Swain, J. Phys. Chem. Solids 31, 1963 (1970).

${ }^{31}$ D. K. Ferry and R. O. Grondin, Physics of Submicron Devices (Plenum, New York, 1991). 
${ }^{32}$ E. Conwell, High Field Transport in Semiconductors (Academic, New York, 1967).

${ }^{33}$ T. Elsaesser, R. Baüerle, and W. Kaiser, Infrared Phys. 29, 503 (1989).

${ }^{34}$ T. Elsaesser, R. J. Baüerle, and W. Kaiser, Phys. Rev. B 40, 2976 (1989).

${ }^{35}$ R. B. James and D. L. Smith, IEEE J. Quantum Electron. 18, 1841 (1982).

${ }^{36}$ B. D. Schwartz, P. M. Fauchet, and A. V. Nurmikko, Opt. Lett. 5, 371 (1980).

${ }^{37}$ M. P. Hasselbeck, Ph.D. thesis, University of Central Florida, 1995 (unpublished).

${ }^{38}$ F. Stern and J. R. Dixon, J. Appl. Phys. 30, 268 (1959).

${ }^{39}$ F. Stern and R. Talley, Phys. Rev. 100, 1638 (1955).

${ }^{40}$ B. S. Wherrett, J. Opt. Soc. Am. B 1, 67 (1984).

${ }^{41}$ H. S. Brandi and C. B. de Araujo, J. Phys. C 16, 5929 (1983).

${ }^{42}$ M. Sheik-Bahae, D. C. Hutchings, D. J. Hagan, and E. W. Van Stryland, IEEE J. Quantum Electron. 27, 1296 (1991).

${ }^{43}$ S. M. Ryvkin, Phys. Status Solidi 11, 285 (1965).

${ }^{44}$ M. P. Hasselbeck, E. W. Van Stryland, and M. Sheik-Bahae (unpublished).

${ }^{45}$ L. V. Keldysh, Sov. Phys JETP 20, 1307 (1965).

${ }^{46}$ C. Cantrell, J. Figueira, J. Scott, and M. O. Scully, Appl. Phys. Lett. 28, 442 (1976).

${ }^{47}$ T. Nee, C. Cantrell, J. Scott, and M. O. Scully, Phys. Rev. B 17, 3936 (1978).
${ }^{48}$ J. Dempsey, J. Smith, G. Holah, and A. Miller, Opt. Commun. 26, 265 (1978).

${ }^{49}$ S. A. Jamison and A. V. Nurmikko, Phys. Rev. B 19, 5185 (1979).

${ }^{50}$ A. L. Smirl, T. F. Boggess, B. S. Wherrett, G. P. Perryman, and A. Miller, IEEE J. Quantum Electron. 19, 690 (1983).

${ }^{51}$ S. W. Kurnick and J. Powell, Phys. Rev. 116, 597 (1959).

${ }^{52}$ The partitioning of excess energy $\Delta E$ between electrons and holes depends on the relative contribution of the light- and heavy-hole band to the three-photon excitation rate.

${ }^{53}$ M. Woerner and T. Elsaesser, Phys. Rev. B 51, 17490 (1995).

${ }^{54}$ M. Sheik-Bahae, P. Mukherjee, and H. S. Kwok, J. Opt. Soc. Am. B 3, 379 (1986).

${ }^{55}$ E. Burstein, Phys. Rev. 93, 632 (1954).

${ }^{56}$ H. J. Hrostowski, G. Wheatley, and W. Flood, Phys. Rev. 95, 1683 (1954)

${ }^{57}$ This formula is valid provided the Fermi energy is high enough in the conduction band to satisfy the inequality $E_{F} \geqslant 3 / 2 k T_{e}$.

${ }^{58}$ J. E. Smith, M. I. Nathan, and J. C. McGroddy, Appl. Phys. Lett. 15, 242 (1969).

${ }^{59}$ C. L. Dick and B. Ancker-Johnson,, Phys. Rev. B 5, 526 (1972).

${ }^{60}$ D. K. Ferry, H. Heinrich, W. Keeler, and E. A. Müller, Phys. Rev. B: Solid State 8, 1538 (1973).

${ }^{61}$ S. Y. Auyang, and P. A. Wolff, J. Opt. Soc. Am. B 6, 595 (1989).

${ }^{62}$ S. Y. Yuen and P. A. Wolff, Appl. Phys. Lett. 40, 457 (1982). 